\title{
Análise experimental dos efeitos da presença de espuma no bombeamento centrífugo de polpa de minério de ferro
}

Reginaldo Amaro Ferreira ${ }^{1 *}$ (D) Aline Pereira Leite Nunes ${ }^{1}$

\section{Resumo}

O bombeamento de espumas geradas nos processos de flotação e deslamagem de minério de ferro, gera grandes dificuldades quando se utiliza bombas centrífugas de polpas. A força centrífuga separa a polpa e o ar, arremessando a polpa para periferia e acumulando o ar no centro do rotor. $\mathrm{O}$ ar aprisionado bloqueia o caminho da polpa causando variações no desempenho hidráulico do sistema. Foi analisado uma instalação de bombeamento de polpa de minério de ferro no Quadrilátero Ferrífero - MG, para verificar os efeitos da presença de espuma no desempenho hidráulico do sistema de bombeamento. Os dados foram coletados durante sessenta dias por instrumentos instalados em campo, pelo sistema de gerenciamento e monitoramento operacional e realização de ensaios em laboratório. As análises revelaram que quando o valor do percentual de espuma na polpa atingiu $14,58 \%$, a vazão volumétrica reduziu cerca de $10 \%$ e a densidade da polpa reduziu $23 \%$. Embora a perda de carga do sistema permanecera inalterada, a altura manométrica total apresentava valor de $36,9 \mathrm{~m}$ inicialmente e $22,6 \mathrm{~m}$ no final, influenciada pela redução dos valores da densidade $\left(1,80\right.$ a $\left.1,21 \mathrm{t} / \mathrm{m}^{3}\right)$. Com a presença de espuma no bombeamento, o NPSHd mostrou valores inferiores ao NPSHr (NPSHr $=2,9 \mathrm{~m}$ e NPSHd $<2 \mathrm{~m}$ ), indicando possibilidade de cavitação. A potência exigida pelo sistema aumentou cerca de $18 \%$ à medida em que o rendimento instantâneo da bomba diminuía, também devido ao aumento de espuma na polpa.

Palavras-chave: Bombeamento de polpa; Bombeamento de espuma; Flotação; Minério de ferro.

\section{Experimental analysis of the effects of froth presence on iron ore slurry centrifugal pumping}

\begin{abstract}
The pumping of froths generated in the iron ore flotation and flaking processes creates great difficulties when using centrifugal slurry pumps. Centrifugal force separates the pulp and air, throwing the pulp to the periphery and accumulating air in the center of the rotor. Trapped air blocks the pulp path causing variations in system hydraulic performance. An iron ore pulp pumping facility was analyzed at Quadrilátero Ferrífero - MG, to verify the effects of the presence of froth on the hydraulic performance of the pumping system. Data were collected for sixty days by field-installed instruments, the operational management and monitoring system and laboratory testing. Analyzes revealed that when the froth percentage value in the pulp reached $14.58 \%$, the volumetric flow rate decreased by about $10 \%$ and the pulp density decreased by $23 \%$.Although the system pressure drop remained unchanged, the total head was $36.9 \mathrm{~m}$ initially and $22.6 \mathrm{~m}$ at the end, influenced by the reduction in density values $\left(1.80\right.$ to $\left.1.21 \mathrm{t} / \mathrm{m}^{3}\right)$. With the presence of froth in the pumping, NPSHd showed lower values than NPSHr (NPSHr $=2.9 \mathrm{~m}$ and NPSHd $<2 \mathrm{~m}$ ), indicating the possibility of cavitation. The power required by the system increased by about $18 \%$ as the instantaneous pump performance decreased, also due to increased froth in the slurry.
\end{abstract}

Keywords: Slurry pumping; Froth pumping; Flotation; Iron ore.

IInstituto de Educação Tecnológica Eireli-IETEC, Belo Horizonte, MG, Brasil.

*Autor correspondente: ramaro.eng@gmail.com

2176-1523 (C) 2021. Ferreira et al. Publicado pela ABM. Este é um artigo publicado em acesso aberto (Open Access) sob a licença Creative Commons Attribution, que permite uso, distribuição e reprodução em qualquer meio, sem restrições desde que o trabalho original seja corretamente citado. 


\section{Introdução}

Nas instalações de beneficiamento de minério de ferro, o transporte de sólidos mais usual é feito em forma de polpa por meio de bombas centrífugas específicas. O minério é adicionado a um fluido de transporte, geralmente a água, e as partículas são mantidas em suspensão [1].

As bombas centrífugas são os equipamentos mais eficientes e econômicos para o transporte de grandes volumes de partículas em suspensão. São frequentemente instaladas em aplicações onde os fluidos viscosos e não-newtonianos devem ser transportados em curtas distâncias [2].

Compreender a reologia da polpa talvez seja o aspecto mais importante a ser considerado no projeto de um sistema de bombeamento. Analisar as suspensões e prever como elas comportam-se quando bombeadas é uma tarefa complexa, especialmente quando se trata de fluidos não-newtonianos contendo grandes quantidades de ar (espumas ou bolhas) e, portanto, não fluem facilmente como a água [3].

As espumas ocorrem naturalmente ou podem ser obtidas intencionalmente durante as operações de beneficiamento mineral. A ocorrência natural pode ser devido à natureza do minério beneficiado, à granulometria e outras propriedades. A obtenção intencional tem a finalidade de separar diferentes minerais por flotação, em que bolhas são criadas pela aeração da polpa por meio da injeção de ar em um processo contínuo [4,5].

Neste trabalho, os resultados de testes experimentais conduzidos com fluídos não-newtonianos em uma bomba centrífuga de grande porte serão apresentados para destacar os efeitos da presença de espuma no desempenho hidráulico da bomba em relação aos dados obtidos com polpa sem espuma. A bomba está instalada em uma planta de beneficiamento de minério de ferro na etapa de deslamagem. Os dados reológicos da polpa foram obtidos por instrumentos instalados na planta e por meios de análises laboratoriais, com o objetivo de certificar a confiabilidade das informações.

\section{Materiais e método}

As análises foram realizadas em uma bomba centrífuga projetada para operar com sólidos em suspensão, acionada por um motor elétrico com potência de $650 \mathrm{cv}$. O acionamento ainda possui um redutor de velocidade que determina a rotação fixa da bomba em $716 \mathrm{rpm}$. O impulsor (rotor) é do tipo aberto com diâmetro de $730 \mathrm{~mm}$ e 8 aletas. A sucção da bomba possui diâmetro de 20 polegadas $(474,5 \mathrm{~mm})$, altura estática (Hs) de 1,5 m e comprimento linear de 3,0 m. Como acessórios ainda possui uma válvula guilhotina pneumática de diâmetro 20 polegadas $(474,5 \mathrm{~mm})$ e uma redução de 20 x 16 polegadas $(474,5 \mathrm{~mm}$ x 406,4 mm). O recalque (descarga da bomba) tem diâmetro de 18 polegadas (427,7 mm) e comprimento total equivalente de $74 \mathrm{~m}$, sendo $30 \mathrm{~m}$ de tubulação linear, 3 curvas de $90^{\circ}$ e o comprimento equivalente (Leq) referente ao distribuidor de polpa. A pressão de alimentação do hidrociclone imediatamente seguinte é de $1,98 \mathrm{kgf} / \mathrm{cm}^{2}$.

Durante o período observado (sessenta dias), a densidade da polpa foi medida por um sensor radiométrico modelo Minitrac 31 do fabricante Vega, instalado na tubulação de descarga da bomba, sendo os valores da densidade confirmados por meio de ensaios em laboratório das 60 (sessenta) amostras coletadas. Os valores da vazão volumétrica foram obtidos pelo medidor de vazão magnético modelo AXF do fabricante Yokogawa instalado na tubulação de descarga da bomba.

Os valores da corrente elétrica do motor, utilizados no cálculo da potência consumida e potência efetiva do sistema de acionamento da instalação de bombeamento, foram obtidos por meio de amperímetros modelo CFW-11 instalados nos quadros de comando dos motores e, assim como os medidores de densidade e vazão da polpa, são interligados ao sistema de monitoramento de variáveis da planta (PIMS).

\section{Resultados e discussões}

$\mathrm{Na}$ instalação de bombeamento observou-se que, quando a polpa bombeada apresentava valores próximos a 5,25\% do volume em espuma, a vazão registrada foi de $1420 \mathrm{~m}^{3} / \mathrm{h}$; com polpa em torno de $14,58 \%$ de espuma (valor máximo registrado), a vazão encontrada foi de $1281 \mathrm{~m} 3 / \mathrm{h}$. Quando o circuito operou com polpa de minério de ferro com percentual de espuma em volume abaixo de $1 \%$, aqui denominado polpa sem espuma, a vazão de polpa aumentou cerca de $80 \mathrm{~m}^{3} / \mathrm{h}$ em relação a vazão máxima encontrada e se manteve constante em aproximadamente $1500 \mathrm{~m}^{3} / \mathrm{h}$. A Figura 1 ilustra o comportamento operacional da vazão volumétrica da polpa de minério de ferro com espuma e sem espuma.

Percentuais de espuma na polpa em torno de 5\%, indicou densidades próximas de $1,57 \mathrm{~g} / \mathrm{cm}^{3}$, enquanto percentuais maiores de espuma, algo em torno de $15 \%$, indicaram valores de densidade bem menores, cerca de $1,21 \mathrm{~g} / \mathrm{cm}^{3}$ (Figura 2).

Os valores variaram de acordo com cada uma das 60 amostras e estão resumidos na Tabela 1.

Tabela 1. Dados gerais das amostras

\begin{tabular}{cccc}
\hline & \multicolumn{3}{c}{ Deslamagem primária } \\
\cline { 2 - 4 } & Amostras & Densidade da polpa t/m & \% sólidos \\
\hline Com espuma & 1 a 60 & 1,21 a 1,56 & 23,55 a 48,16 \\
Sem espuma & 1 a 60 & 1,79 a 1,81 & 49,01 a 51,94 \\
\hline
\end{tabular}




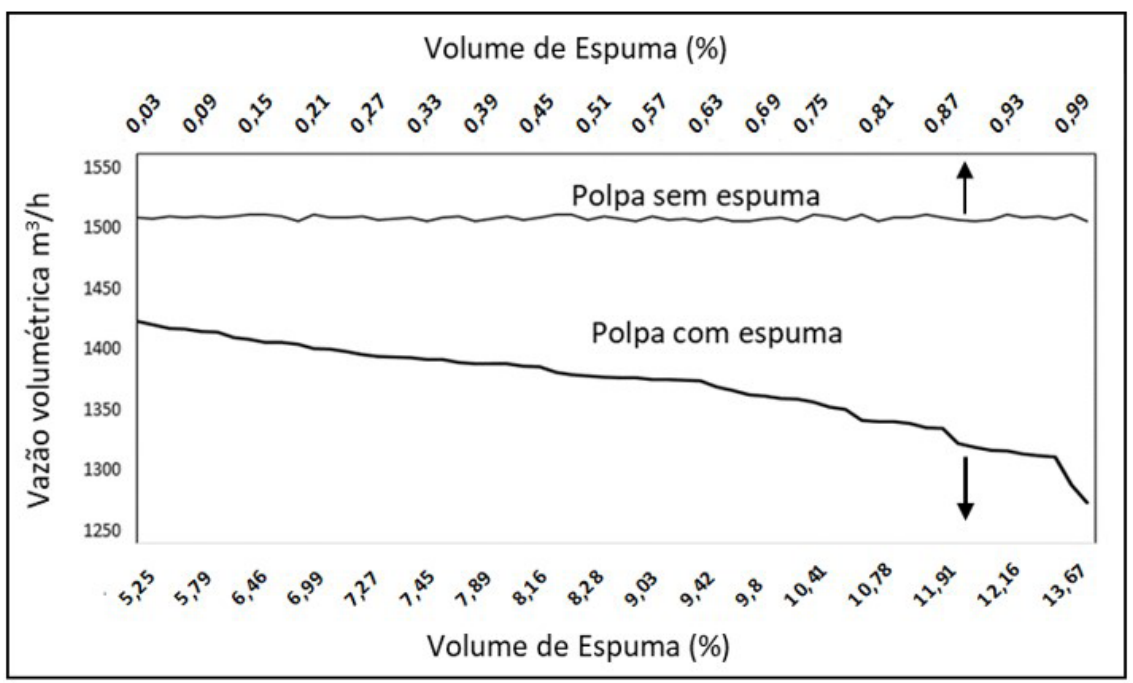

Figura 1. Relação entre vazões (polpa com espuma e sem espuma).

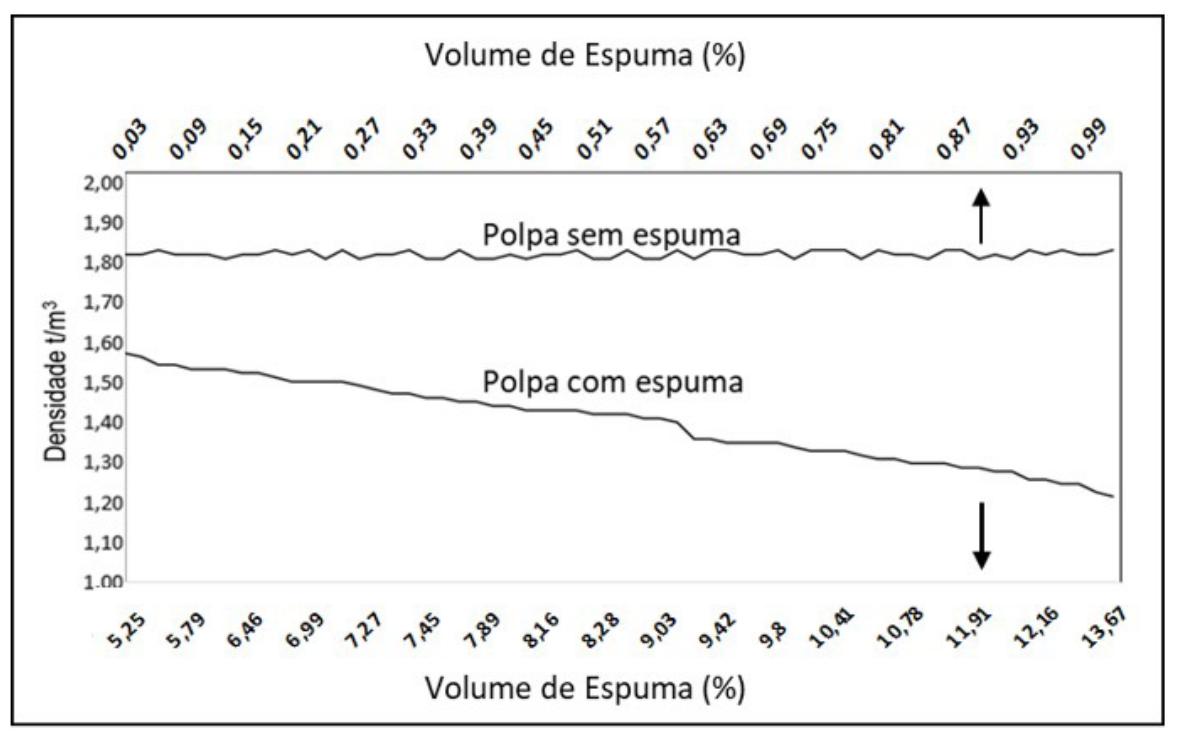

Figura 2. Relação entre espuma e densidade.

Adeterminação da densidade das amostras em laboratório foi útil para comparação com os valores obtidos no medidor de densidade instalado na tubulação de recalque da bomba. Quando comparamos os valores da densidade resultantes dos ensaios laboratoriais e os valores da densidade obtidos pelo densímetro instalado no campo modelo MINITRAC 31, observa-se que não houveram diferenças consideráveis entre as duas medições. $\mathrm{O}$ desvio padrão entre os dois resultados foi inferior a $1 \%$.

A presença de espuma também teve forte influência na viscosidade da polpa. As medições ocorridas em laboratório demonstraram que o aumento da quantidade de espuma na polpa diminuiu consideravelmente a sua viscosidade. Amostras de polpa sem espumas apresentaram valores de viscosidade em torno de $10,93 \mathrm{cP}$ e amostras de polpa com cerca de $14 \%$ de espuma em volume, apresentaram valores de viscosidade em torno de $2,53 \mathrm{cP}$.
A Figura 3 ilustra o comportamento da viscosidade da polpa em função da presença de espuma na polpa. A polpa sem espuma demonstrou valores de viscosidade maiores, que variaram de 9,45 cP a 10,93 cP. Por outro lado, a polpa com espuma apresentou um comportamento contrário. À medida em que o volume de espuma na polpa aumentava a viscosidade da mistura diminuía consideravelmente, com valores que variaram de $2,53 \mathrm{cP}$ a $6,88 \mathrm{cP}$.

$\mathrm{Na}$ instalação de bombeamento onde o experimento foi realizado, o NPSHr informado pelo fabricante é de 2,9 m e o NPSHd pela instalação é de 7,2 m. Porém, foi observado que quando há excesso de espuma na sucção da bomba (percentuais acima de 5\% de ar em forma de bolhas), o NPSHd da instalação reduz e o NPSHr aumenta (Figura 4). Esse descontrole operacional influencia diretamente na presença do fenômeno de cavitação e o resultado na prática 


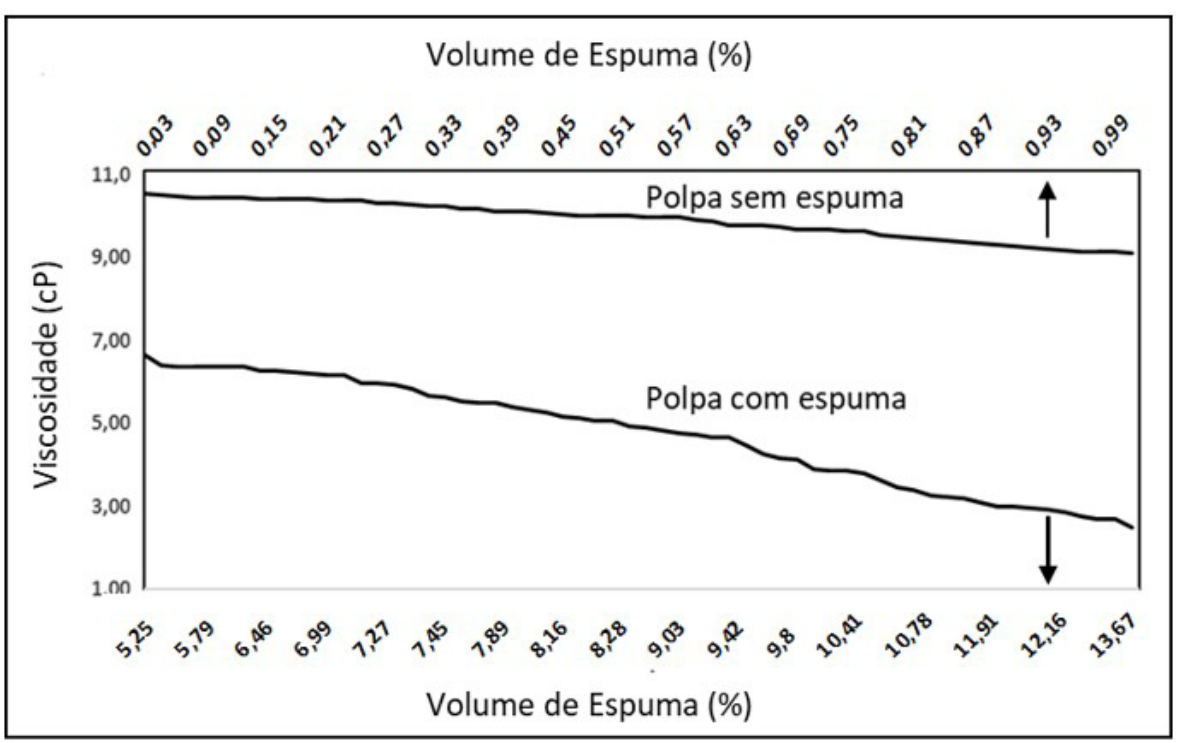

Figura 3. Relação entre viscosidade e variação do volume de espuma.

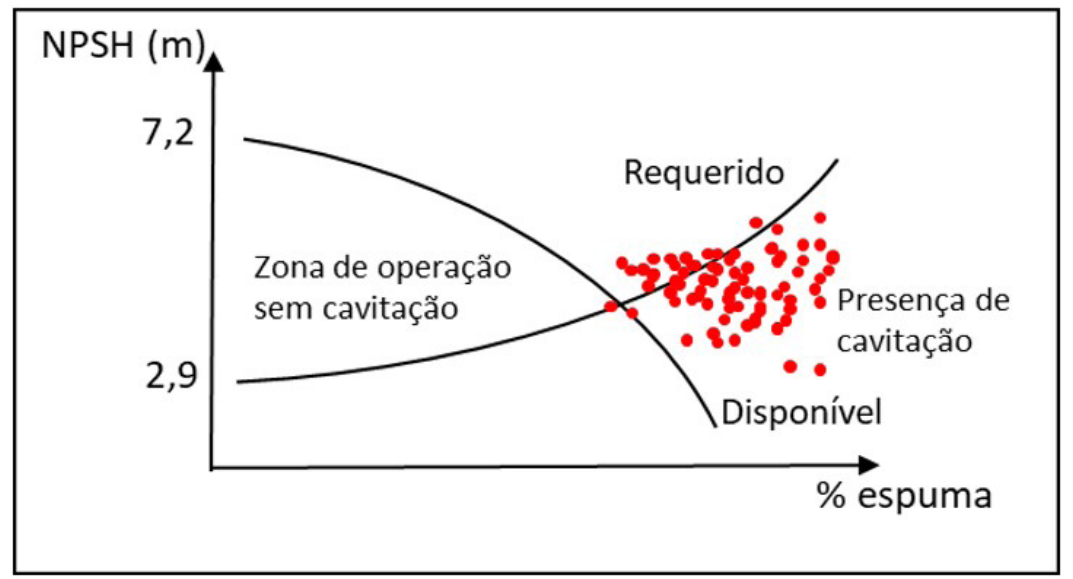

Figura 4. NPSH com presença de espuma na sucção da bomba.

é o desgaste do rotor, ocasionando baixo rendimento da bomba e redução da capacidade de bombeamento.

Utilizou-se a Equação 1 geral de cálculo da potência no bombeamento apresentada por Addie et al. [6] no padrão ANSI/HI 12.1-12.6-2007 do Hydraulic Institute Standards. Notou-se que a potência calculada para acionamento do sistema sem a consideração de espumas, ficou estável em $550 \mathrm{cv}$, mas quando adicionou-se espumas ao bombeamento, a densidade da polpa de minério de ferro diminuiu, reduzindo os valores da potência calculada para a faixa entre 300 e $450 \mathrm{cv}$ (Figura 5).

$$
\mathrm{N}=\frac{Q^{*} \gamma * \text { Hman }}{\eta}
$$

Nesta equação, a potência requerida no sistema $(\mathrm{N})$ é calculada a partir dos valores da vazão desejada (Q), dos valores encontrados da densidade da mistura $(\gamma)$, da altura manométrica (Hman) e do rendimento hidráulico da bomba.

A potência efetiva, que é compreendida pela energia que realmente realiza trabalho, é a energia consumida pela instalação de bombeamento para transportar a polpa (Equação 2).

$$
P_{e}=I * V * \sqrt{3} * \cos \varphi
$$

O valor da tensão elétrica é conhecido no sistema e é fixo. Nesta instalação o motor de acionamento da bomba é um motor de $4000 \mathrm{v}$ (volts). O coeficiente $\sqrt{3}$ também é fixo, pois trata-se de um motor elétrico trifásico. Já o fator de potência $(\operatorname{Cos} \varphi)$ varia de equipamento para equipamento, que é sempre fornecido pelo fabricante. Nesta instalação o motor de acionamento possui um fator de potência igual a $86 \%$ (Figura 6). 


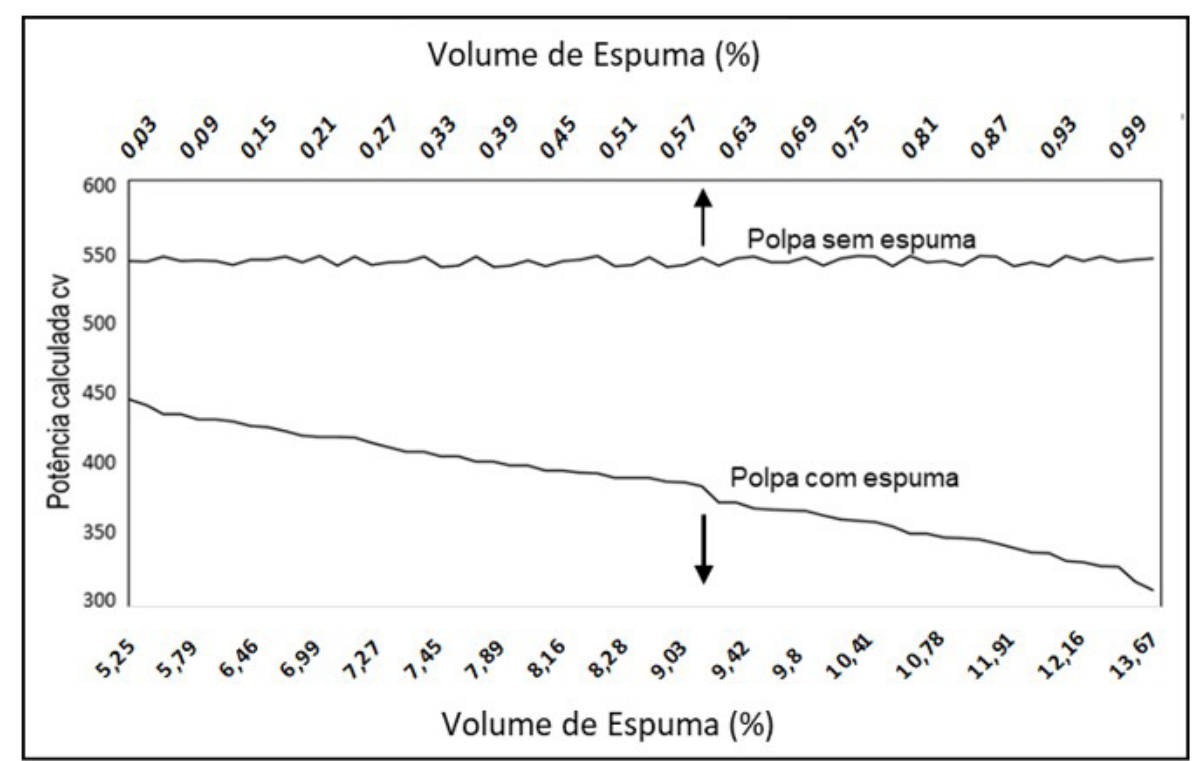

Figura 5. Relação entre espuma e potência calculada.

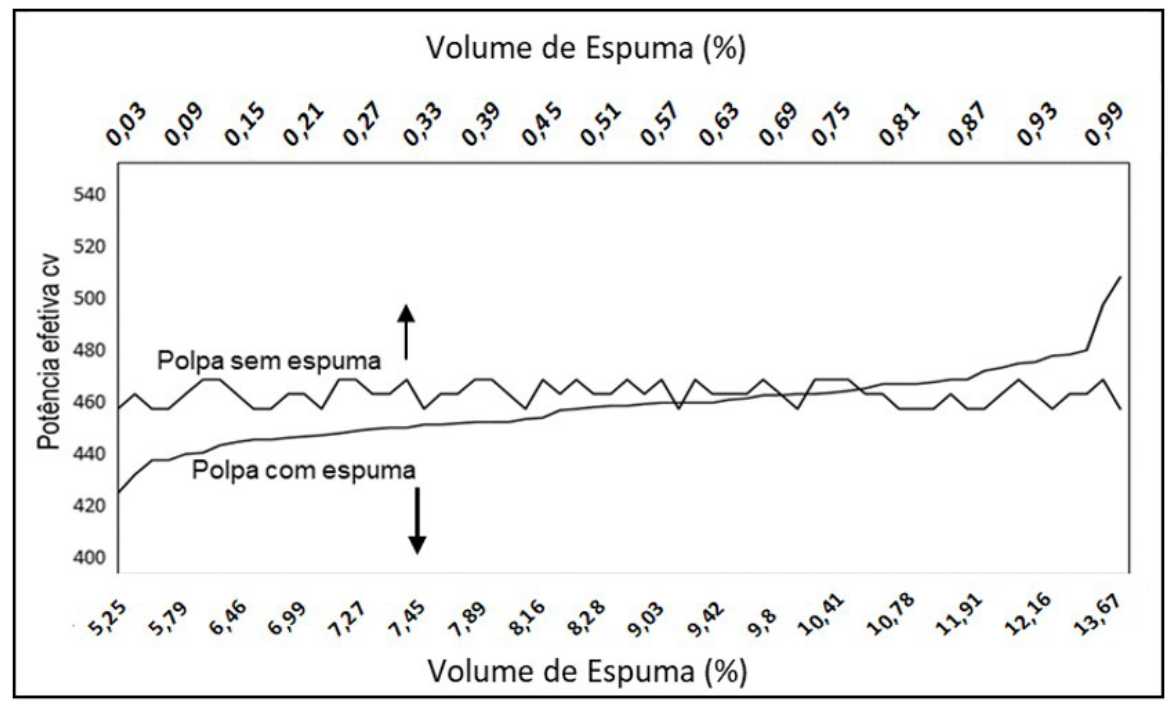

Figura 6. Potência efetiva do sistema.

A espuma tem facilidade em segregar-se em zonas de densidades diferentes no bocal de sucção da bomba. $\mathrm{O}$ ar tem uma propensão para se separar da polpa e é succionado pelo centro do rotor, bloqueando o fluxo de entrada da polpa de minério de ferro. Para vencer a resistência criada pelo bloqueio do fluxo de polpa pelo ar presente na espuma, o motor é solicitado a fornecer mais energia para o sistema, elevando a sua corrente elétrica (I).

A partir deste fenômeno é possível afirmar que, nesta instalação estudada, quanto maior for o percentual de espuma, variando entre 0 e 14,58\% (valores encontrados), maior será a potência efetiva do sistema e maiores serão os valores da energia para realizar o trabalho. Confirmando esta tendência, os estudos desenvolvidos pela Weir Minerals [7,8], mostraram que as potências obtidas de uma bomba adaptada para espuma foram superiores às potências obtidas em um sistema com uma bomba específica para o transporte de espuma (Figura 7). No estudo, a potência efetiva aumentou $18 \%$ em relação ao sistema operando apenas com polpa (sem a presença de espuma). A bomba específica para espuma configurava-se com rotor semiaberto e com palhetas de indução de fluxo.

O dimensionamento clássico de bombas para polpa de minério de ferro não considera a presença de espuma como um fator relevante. Na maioria das vezes assume-se o valor da densidade da polpa formada apenas pelo fluido de transporte e o minério. $\mathrm{O}$ acúmulo de ar no interior da bomba provocado pela presença da espuma na polpa causa o baixo desempenho do bombeamento com ciclos de altas e baixas vazões, levando a baixa produção e/ou transbordamento das caixas de alimentação. Por isso é comum os projetistas superdimensionarem as bombas com 


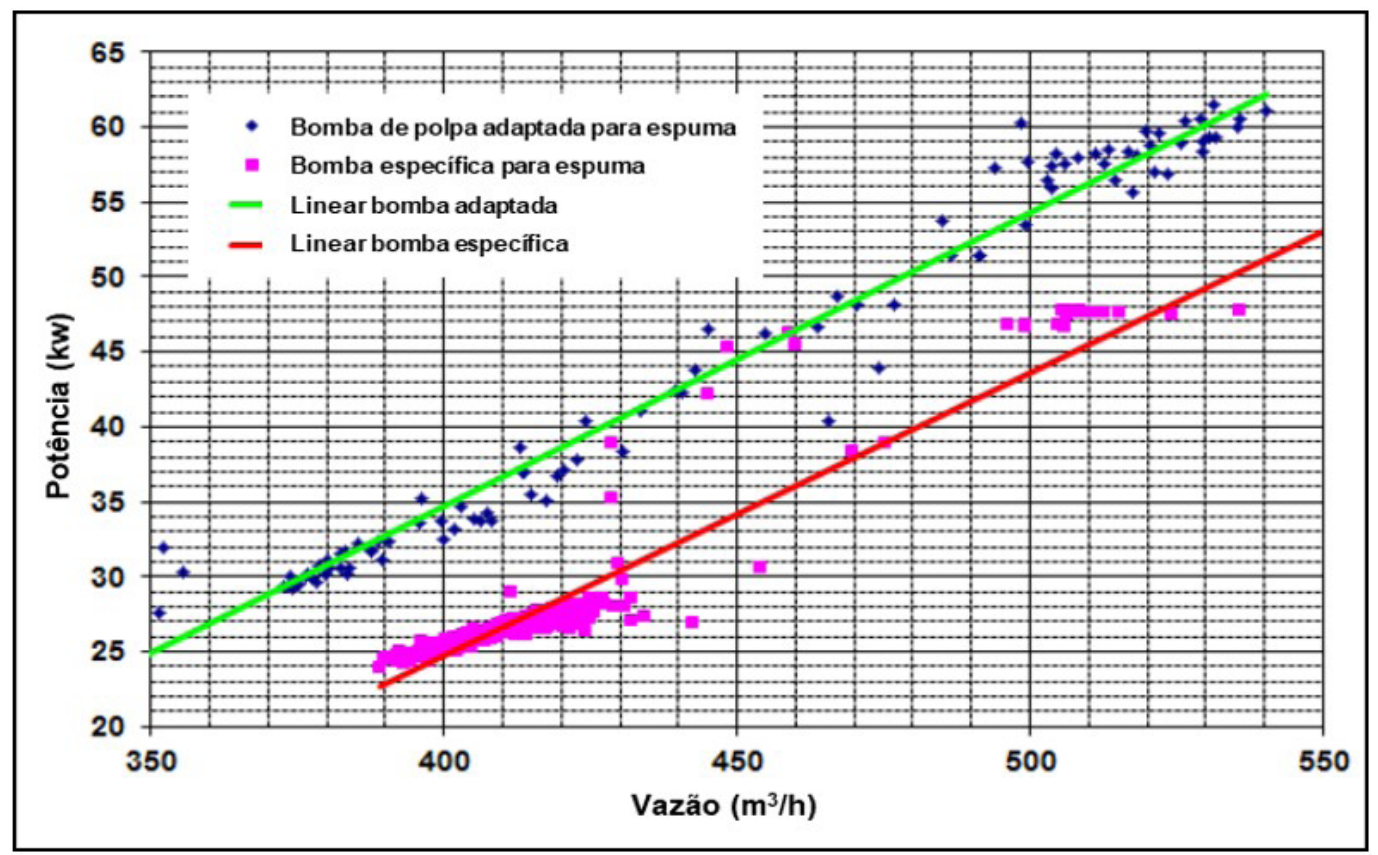

Figura 7. Comparativo de absorção de potência. Fonte: Weir Minerals [7,8]. (Adaptado pelo autor).

o objetivo de absorver os efeitos causados pela presença de espumas na polpa bombeada.

O avanço tecnológico, sobretudo na área da informática, o desenvolvimento de softwares inteligentes e de computadores superpotentes, possibilitam nos dias atuais técnicas de simulações de sistemas de bombeamento antes mesmo de sua instalação, tornando o dimensionamento e seleção dos equipamentos e acessórios mais ágeis, econômicos e confiáveis.

Como contribuição, este trabalho salienta a importância do correto estudo reológico da polpa a ser transportada por bombeamento centrífugo. Uma mistura aerada, caracterizada pela presença de espuma na polpa de minério de ferro em diferentes etapas do processo de beneficiamento, provoca o baixo desempenho hidráulico da instalação de bombeamento, altos custos de operação e manutenção e perdas irreversíveis para produção.

\section{Conclusão}

A análise comparativa do bombeamento de minério de ferro mostrou que a vazão volumétrica diminuiu cerca de 10\% quando o percentual volumétrico de espuma na polpa atingiu valores de $14,58 \%$. O ar em forma de bolhas era liberado e se acumulava no interior da bomba, bloqueando o fluxo da polpa.

A densidade da polpa de minério de ferro diminuiu $23 \%$ quando o percentual de espuma aumentou para $14,58 \%$ na polpa. A densidade antes formada pelo fluido de transporte (água) e o minério de ferro, ganha o ar como mais um componente e torna a mistura mais "leve".
A viscosidade da polpa também teve forte influência da presença de espumas. Com percentuais de espumas próximos a $0 \%$, foram encontrados valores de viscosidade em torno de 10,93 cP. Com percentuais de espumas próximos a $14,58 \%$, a viscosidade da polpa teve valores de $2,53 \mathrm{cP}$.

O NPSH disponível da instalação em vários momentos foi menor que o NPSH requerido (2,9 m). Neste experimento, a instalação disponibilizava um NPSH de $7,2 \mathrm{~m}$, porém com valores percentuais de espuma variando entre $5,25 \%$ e $14,58 \%$, este valor de NPSHd reduziu para valores inferiores a 2,0 m. Tal comportamento propiciou o surgimento de indícios de cavitação e, como consequência, o baixo rendimento hidráulico e o desgaste prematuro dos componentes internos da bomba.

A potência exigida pelo sistema aumentou $18 \%$ à medida que o rendimento instantâneo da bomba variava. $\mathrm{O}$ aumento do percentual de espuma com o consequente acúmulo de ar no interior da bomba bloqueando o fluxo da polpa, passou a exigir do sistema mais energia para vencer a resistência oferecida pelo excesso de ar comprimido. Este aumento da potência efetiva foi detectado pelo aumento da corrente elétrica do motor de acionamento.

\section{Agradecimentos}

Os autores agradecem a todos que contribuíram para a realização do trabalho, em especial ao Instituto de Educação Tecnológica Eireli - IETEC. 


\section{Referências}

1 Ferreira EE, Brandão PRG, Klein B, Peres AEC. Reologia de suspensões minerais: uma revisão. Revista Escola de Minas. 2005;(58):83-87.

2 Taylor WR. A new froth pump for improved pumping of viscous thickener underflow. In: The Southern African Institute of Mining and Metallurgy. Anals of The 6th International Platinum Conference, "Platinum-Metal for the Future"; 201420 January-23 October; Johannesburg. Johannesburg: SAIMM. 2014.

3 Bisco APR. A influência de algumas variáveis sobre a reologia de polpas minerais [disseertação]. Belo Horizonte: Universidade Federal de Minas Gerais; 2009.

4 Li C, Saeed F, Fengnian S, Kym R. A novel approach to measure froth rheology in flotation. Minerals Engineering. 2015;71:89-96.

5 METSO Minerals. Diretrizes básicas para o bombeamento de polpas. Apresentando o software para o dimensionamento de bombas - Metso PumpDim ${ }^{\mathrm{TM}}$ para Windows ${ }^{\mathrm{TM}}$. 1. ed. Suécia: Metso; 2012.

6 Addie GR, Roudnev AS, Sellgren A. The new ANSI/HI centrifugal slurry pump standard. Journal of the Southern African Institute of Mining and Metallurgy. 2007;107:403-409.

7 Weir Minerals. Relatório de adequação à eficiência de bombeamento com relação à presença de espuma. Quadrilátero Ferrífero, Minas Gerais, Brasil, 2016.

8 Weir Minerals. Technical Bulletin, number 28: Froth pumping. Artarmon: Weir Minerals Australia; 2020.

Recebido em: 13 Ago. 2019

Aceito em: 09 Mar. 2020 